\title{
Development of a Retractor Holding Device to Reduce the Manpower in Total Hip Arthroplasty through Direct Anterior Approach
}

\author{
Hirotake Yo, Hirotsugu Ohashi, Ryo Sugama, Tessyu Ikawa \\ Department of Orthopaedic Surgery, Osaka Saiseikai Nakatsu Hospital, Osaka, Japan \\ Email: yangyang1968@hotmail.com
}

Received 13 January 2016; accepted 21 February 2016; published 26 February 2016

Copyright (C) 2016 by authors and Scientific Research Publishing Inc.

This work is licensed under the Creative Commons Attribution International License (CC BY). http://creativecommons.org/licenses/by/4.0/

\section{(c) (i) Open Access}

\begin{abstract}
Direct anterior approach (DAA) for total hip arthroplasty (THA) is a minimally invasive technique. In this approach, two assistants are necessary. We developed a retractor holding device called "Spider arm" to replace an assistant in the contra-lateral side. In this study, we investigated the usefulness of Spider arm in THA through direct anterior approach. 20 hips were operated without Spider arm and 21 hips were operated with Spider arm. The surgery time and the blood loss were compared. The cup position and leg length discrepancy were measured on radiography. There was no statistical significance between two groups in all parameters. No clinical complications were reported. With Spider arm, DAA-THA could be performed by two surgeons without deterioration of the surgery time and blood loss. The accuracy of cup position and leg length discrepancy was not affected. Spider arm can contribute to reducing the manpower in DAA-THA.
\end{abstract}

\section{Keywords}

Retractor Holding Device, Spider Arm, Direct Anterior Approach, Hip Arthroplasty

\section{Introduction}

Direct anterior approach (DAA) for total hip arthroplasty (THA) is a minimally invasive technique [1]-[4] that requires no detaching of muscles or tendons. In this approach, a patient lies in a supine position and two assistants are necessary: the $1^{\text {st }}$ assistant in the operative side and the $2^{\text {nd }}$ assistant in the contra-lateral side.

The main role of the $2^{\text {nd }}$ assistant is to keep holding the retractors. However, this assistant has difficulty in 
seeing the surgical site. When a young surgeon takes this position, it is not optimal from educational point of view. It is better for young surgeon to be in the operative side to learn surgical procedure. And when the hospital is short of orthopedic surgeons, it is difficult to operate DAA-THA with two assistants.

For these reasons, we developed a retractor holding device called "Spider arm" to replace the $2^{\text {nd }}$ assistant in the contra-lateral side. Spider arm has universal joint that provides three dimensions of freedom. The connector can easily reach the retractor and hold it in one action.

In this study, we investigated the advantages and disadvantages of this device in terms of surgery time, blood loss, cup position and leg length discrepancy by comparing the operation with this device to that without it.

\section{Materials and Methods}

Spider arm consists of a pole, a rod, and two curved arms with connectors (Figure 1). A pole is fixed to the side bar in operative table on the contra-lateral side. After draping, sterilized rod is fixed to the pole over the drape. The rod crosses over the pelvis at anterior superior iliac spine. Two curved arms are attached to the rod with universal fixators, and the connectors on the arms link with the retractors (Figure 2). The universal fixator has 90 degrees of freedom in three dimensions, thus the retractor can be held at the optimal position (Figure 3, Figure 4). This wide range of freedom enables us to apply it to various heights and physiques of patients.

In acetabular exposure, the short arm holds the retractor at the anterior edge of the acetabular, and the long arm holds the retractor at obturator foramen (Figure 5, Figure 6). The assistant holds only one retractor at the posterior edge of the acetabulum. In femoral exposure, the short arm holds the retractor at the medial side of the femur (Figure 7). The assistant hold the retractor at greater trochanter to elevate the femur and hold the patient's leg in adduction and external rotation.

From January to June 2012, 20 hips were consecutively operated without Spider arm with an average age of 66.8 years old at time of surgery (range 60 - 84 years old). There were 1 man and 19 women. The average height was $150 \mathrm{~cm}$ (range 144 - 158), and the average BMI was 24.8 (range 21.4 - 35.1). Etiologies were osteoarthritis in 14 hips, idiopathic osteonecrosis in 2 hips and rapid destructive coxarthritis in 4 hips.

From July to December 2012, twenty-one hips were consecutively operated with the device with an average age of 69.4 years old at time of surgery (range 60 - 82 years old). There were 3 men and 18 women. The average

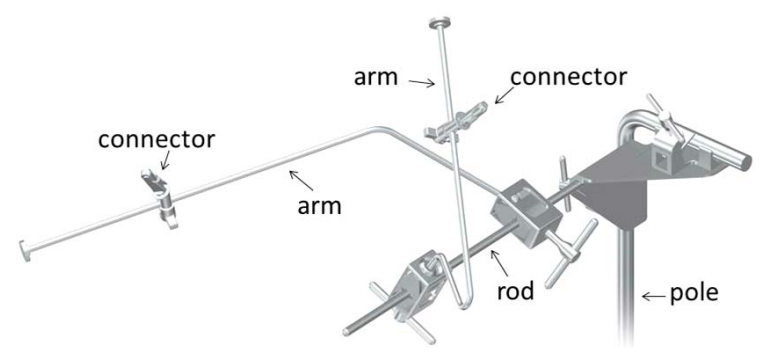

Figure 1. Structure of Spider arm.

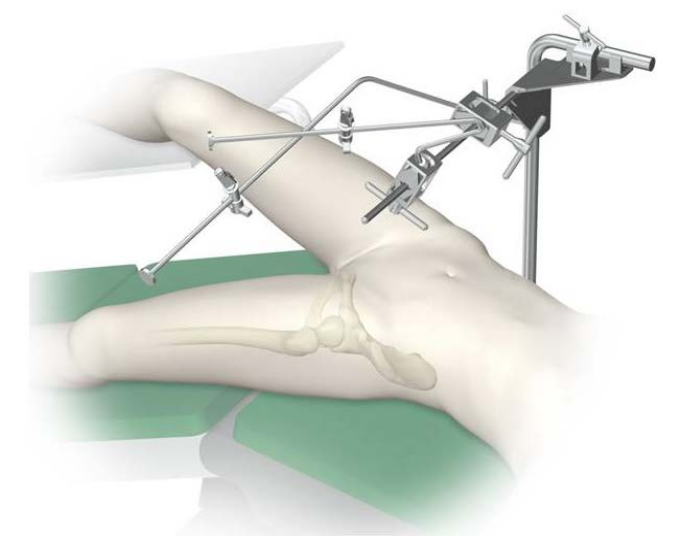

Figure 2. Setting of Spider arm in DAA-THA. 


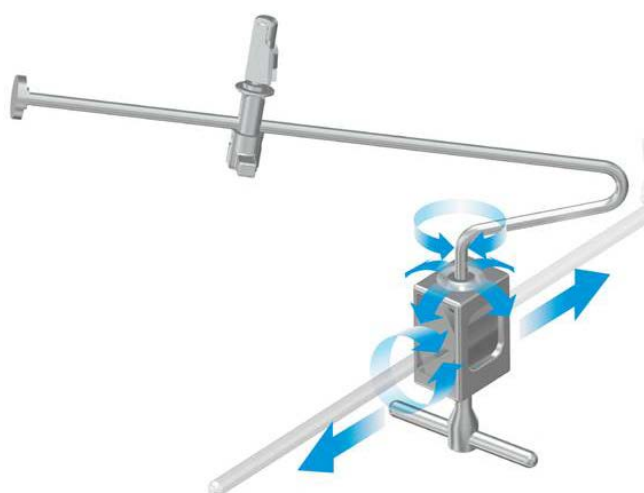

Figure 3. Mobility of universal fixator.

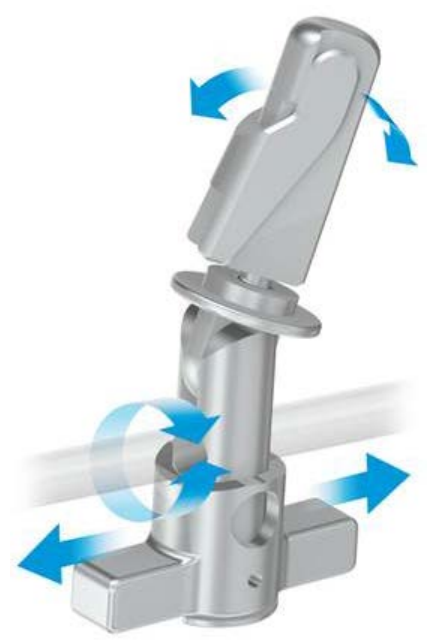

Figure 4. Mobility of connector.

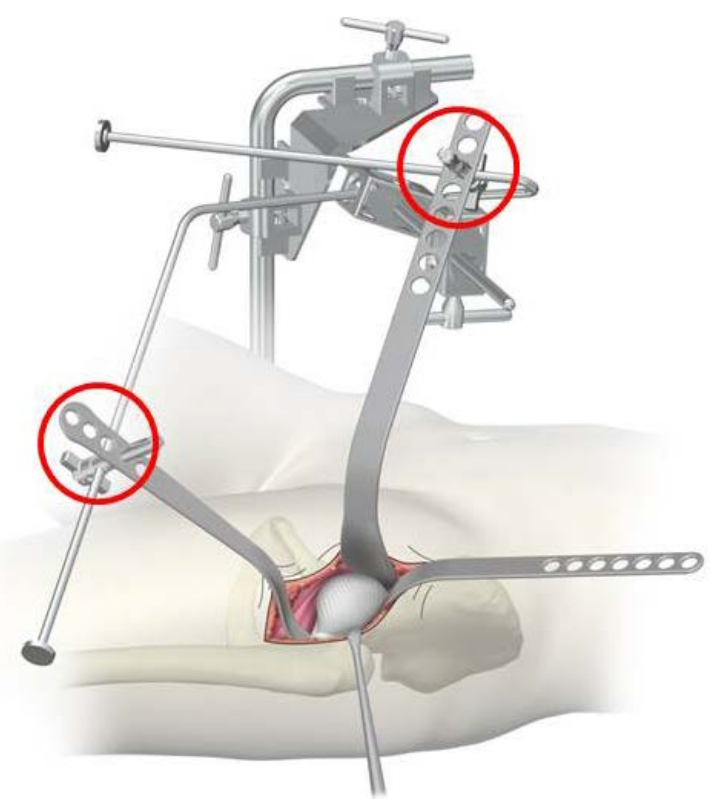

Figure 5. Setting of retractors with Spider arm in capsule exposure. 

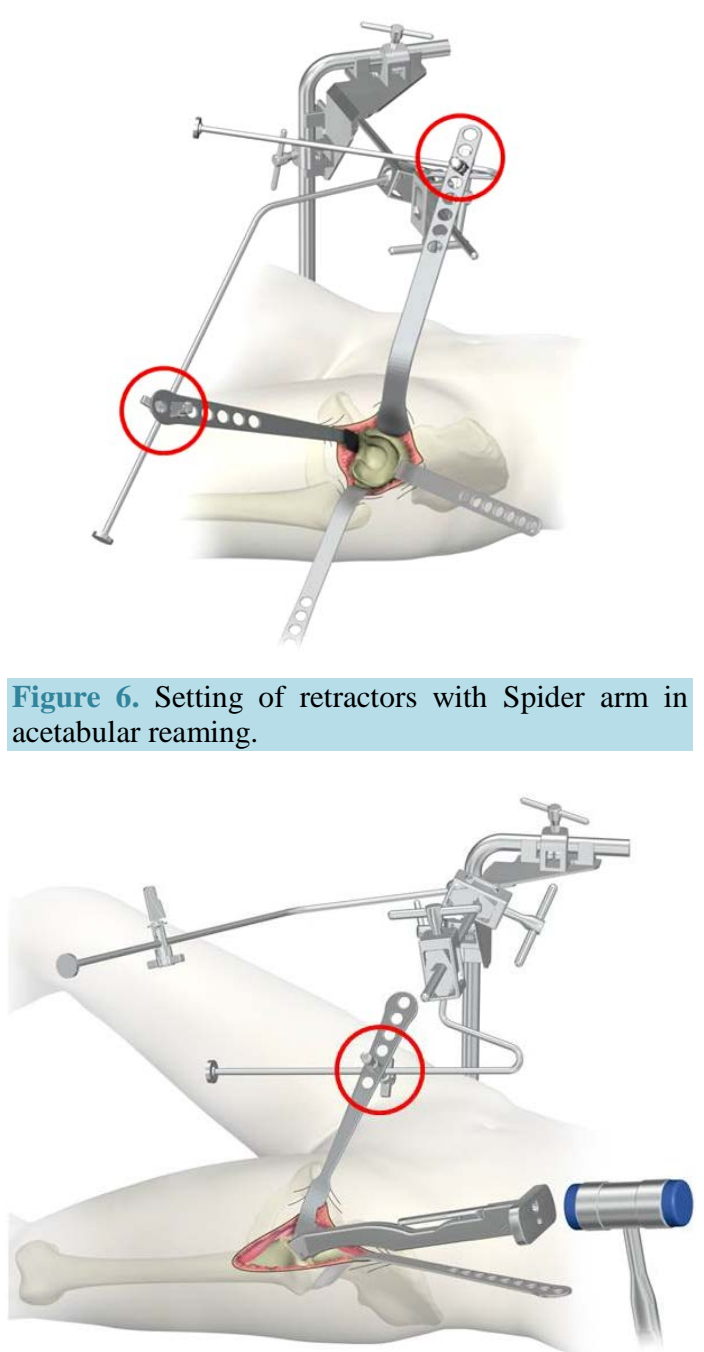

Figure 7. Setting of retractors with Spider arm in femoral rasping.

height was $154 \mathrm{~cm}$ (range 142 - 171), and the average BMI was 22.3 (range 20.4 - 27.1). Etiologies were osteoarthritis in 15 hips, idiopathic osteonecrosis in 4 hips and femoral neck fracture in 2 hips. To assess for differences in demographic characteristics of the study population between groups, student's t-test was performed. A p-value of $<0.05$ was taken to indicate statistical significance (Table 1 ).

In each group, the surgery time, the blood loss was recorded. The cup position and leg length discrepancy were measured on radiography. To assess for difference in each parameters between groups, student's t-test was performed. A p-value of $<0.05$ was taken to indicate statistical significance. And intraoperative complications [5]-[7] were recorded.

The study was conducted after patients signed informed consent.

\section{Results}

The average surgery time was $122 \pm 18$ minutes without Spider arm and $129 \pm 16$ minutes with Spider arm. The average blood loss was $477 \pm 196 \mathrm{~g}$ and $397 \pm 172 \mathrm{~g}$, respectively. The cup abduction angle and anteversion were $41.7 \pm 5.6$ degrees and $12.9 \pm 3.9$ degrees without Spider arm, and $40.9 \pm 5.1$ degrees and $13.7 \pm 3.5$ degrees with Spider arm. The leg length discrepancy was $0.85 \pm 3.7 \mathrm{~mm}$ and $1.85 \pm 2.9 \mathrm{~mm}$, respectively. There was no statistical significance between two groups in all parameters (Table 2). No clinical complications were reported in each group. 
Table 1. Demographic data.

\begin{tabular}{cccc}
\hline & With Spider arm & Without Spider arm & $\begin{array}{c}\text { Student's t-test } \\
(\mathrm{P}<0.05)\end{array}$ \\
\hline Gender & Male 3; Female 18 & Male 1; Female 19 & \\
Age & $69.4(60-82)$ & $66.8(60-84)$ & n.s. \\
Height $(\mathrm{cm})$ & $154(142-171)$ & $150(144-158)$ & n.s. \\
Weight $(\mathrm{kg})$ & $53.7(40-75)$ & $53.9(42-79)$ & n.s. \\
BMI & $22.3(20.4-27.1)$ & $24.4(21.4-35.1)$ & n.s. \\
\hline
\end{tabular}

Table 2. Differences in operation.

\begin{tabular}{cccc}
\hline & With Spider arm & Without Spider arm & $\begin{array}{c}\text { Student's t-test } \\
(\mathrm{p}<0.05)\end{array}$ \\
\hline Surgery time (minutes) & $129 \pm 16$ & $122 \pm 18$ & n.s. \\
Blood loss (ml) & $397 \pm 172$ & $477 \pm 196$ & n.s. \\
Cup abduction angle (degree) & $40.9 \pm 5.1$ & $41.7 \pm 5.6$ & n.s. \\
Cup anteversion (degree) & $13.7 \pm 3.5$ & $12.9 \pm 3.9$ & n.s. \\
\hline
\end{tabular}

\section{Discussion}

Orthopedic surgeons use several instruments or devices to assist THA through DAA. For example, chain and weight pull the retractor. Ring or u-shaped bar connects the retractor. Compared to these instruments, our device can hold the retractor at the optimal position. Magic tower was originally developed for general surgery. Recently, some surgeons use Magic tower for THA and reported its efficacy [8] [9]. Compared to Magic tower, Spider arm is compact and easy to handle. As it is less space occupying, it does not disturb the operative procedures. The wide range of freedom enables to hold the retractor in proper direction with appropriate traction. The operator can fix or release the arm in one action.

Since Spider arm replaces the task of the $2^{\text {nd }}$ assistant in the contra-lateral side, THA through DAA can be performed by two surgeons instead of three. Though there might be the possibility to take times to set Spider arm and to set retractors to the Spider arm, the surgery time were not deteriorated when operated by two surgeons with Spider arm. The accuracy of cup position and the correction of leg length discrepancy were not affected. So, there seems to be no disadvantage to use Spider arm.

Femoral nerve palsy is a major complication of THA through DAA. One of the causes is over-traction of the retractor which is placed at the anterior edge of acetabulum. Sometimes the $2^{\text {nd }}$ assistant holds the retractor so extremely to see the operative site, and the risk of femoral nerve palsy will increase. In contrast, Spider arm holds the anterior retractor under surgeon's control, and the over-traction can be avoided. This point might be an advantage of using Spider arm.

From these results, Spider arm can replace the task of $2^{\text {nd }}$ assistant, and the operation was performed safely without any deterioration.

\section{Conclusion}

With Spider arm, THA through DAA can be performed by two surgeons without any deterioration in terms of surgery time and blood loss. The accuracy of cup position and leg length correction was not affected. Spider arm can contribute to reducing the manpower in THA through DAA.

\section{References}

[1] Post, Z.D., Orozco, F., Diaz-Ledezma, C., Hozack, W.J. and Ong, A. (2014) Direct Anterior Approach for Total Hip 
Arthroplasty: Indications, Technique, and Results. Journal of the American Academy of Orthopaedic Surgeons, 22, 595-603. http://dx.doi.org/10.5435/JAAOS-22-09-595

[2] Berend, K.R., Lombardi Jr, A.V., Seng, B.E. and Adams, J.B. (2009) Enhanced Early Outcome with the Anterior Supine Intermuscular Approach in Primary Total Hip Arthroplasty. The Journal of Bone \& Joint Surgery, 91, 107-120. http://dx.doi.org/10.2106/JBJS.I.00525

[3] Kennon, R.E., Keggi, J.M., Wetmore, R.S., Zatorski, L.E., Huo, M.H. and Keggi, K.J. (2003) Total Hip Arthroplasty through a Minimally Invasive Anterior Surgical Approach. The Journal of Bone \& Joint Surgery, 85, 39-48.

[4] Kennon, R., Keggi, J.M., Zatorski, L.E. and Keggi, K.J. (2004) Anterior Approach for Total Hip Arthroplasty: Beyond the Minimally Invasive Technique. The Journal of Bone \& Joint Surgery, 86, 91-97.

[5] Goulding, K., Beaule, P.E., Kim, P.R. and Fazekas, A. (2010) Incidence of Lateral Femoral Cutaneous Nerve Neuropraxia after Anterior Approach Hip Arthroplasty. Clinical Orthopaedics and Related Research, 468, 2397-2404. http://dx.doi.org/10.1007/s11999-010-1406-5

[6] Grob, K., Manestar, M., Ackland, T., Filgueira, L. and Kuster, M.S. (2015) Potential Risk to the Superior Gluteal Nerve during the Anterior Approach to the Hip Joint: An Anatomical Study. The Journal of Bone \& Joint Surgery, 97, 1426-1431. http://dx.doi.org/10.2106/JBJS.O.00146

[7] Farrell, C.M., Springer, B.D., Haidukewych, G.J. and Morrey, B.F. (2005) Motor Nerve Palsy Following Primary Total Hip Arthroplasty. The Journal of Bone \& Joint Surgery, 87, 2619-2625. http://dx.doi.org/10.2106/JBJS.C.01564

[8] Hisakado, H. (2012) Does “Magic Tower” Reduce Surgical Assistants Requirement in Total Hip Arthroplasty through a Direct Anterior Approach? Hip Joint, 38, 342-346 (in Japanese).

[9] Hisakado, H., Kanekasu, K., Nanbu, K. and Hado, Y. (2011) Efficacy of Retractor Holder (Magic Tower) in DAATHA. The Japanese Society for Replacement Arthroplasty, 41, 592-593 (in Japanese). 